\title{
Modeling and Identification of Discrete Second-Order Systems via Stochastic Mechanics
}

\author{
AKIRA OHSUMI \\ Professor Emeritus of Kyoto Institute of Technology, \\ 56 Nakanoda-cho, Ichijoji, Sakyo, Kyoto 606-8163, Japan \\ e-mail: akiraspika@nifty.com
}

\begin{abstract}
For a class of second-order mechanical or structural systems subjected to random disturbances, their discrete-time models are derived. Since the output of stochastic system is random and has no longer its velocity in the ordinary sense, the notion of the Nelson process which is a stochastic process having forward and backward mean velocities is introduced to describe the action integral. Then the stochastic version of the Euler-Lagrange equation is derived from the Hamilton's principle. The discrete-time second-order model is obtained by discretizing the stochastic Euler-Lagrange equation and/or Hamilton's principle. The structural matrices are identified using the discrete second-order model.
\end{abstract}

Keywords: Stochastic system, discrete second-order system, Nelson process, stochastic Euler-Lagrange equation, subspace identification.

\section{Introduction}

The identification of structural parameters such as mass, stiffnes and damping of multidimensional mechanical or strutural systems whose dynamics are described by the vector second-order differential equations (see (3) below) is nowadays one of the most important problems. Especially, in the architectural fields, it is said that the investigation of dynamic characteristics of an existing building structure by field tests is a necessary and important task in the course of checking the construction quality, validating or improving analytical structural models, or conducting damages. The ambient vibration experimental test is considered as the most popular one because of its portability and ease of setup. Up to the present time, the identification problem of the structural parameters has been investigated by many researchers, for instance, refer [1]-[6].

The identification of the structural parameters $((M$, $C, K)$ in (3)) has been traditionally investigated using the first-order continuous-time state-space model, $\dot{x}(t)=A x(t)+B w(t)$, where $w(t)$ is an external input, which is transformed equivalently to the discrete-time system: $x(k+1)=F x(k)+G_{0} w(k)$. Since all structural parameters are shut in the matrix $A$, in order to recover them from the estimates of the matrices $F$ and $G_{0}$, we need to invert the relations, $F=e^{A h}$ and $G_{0}=\left[\int_{0}^{h} e^{A \tau} d \tau\right] B$ where $h(>0)$ is a constant sampling interval. This, as it were, central dogma in the subspace method is the architectonic prop of the d2c routine in MATLAB. However, the recovery of $A$ from the estimated $F$ involves the computation of the logarithm of $F$. As pointed out in [7], for a sufficiently small $h$ the condition number of computing $A$ from $F$ tends to infinity, because $F \rightarrow I$ (unit matrix) and $G_{0} \rightarrow 0$ as $h \rightarrow 0$ so that the discrete-time model becomes degenerated. Hence, the discrete-time model obtained as mentioned above becomes ill-conditioned [7]. Furthermore, the structural matrices, mass $M$, damper $C$ and stiffness $K$, are involved in the forms of $-M^{-1} K$ and $-M^{-1} C$ in the matrix $A$. This serious fact means that each structural matrix can not be obtained separately.

Recently, Bruschetta, Picci and Saccon [8] have roused the notice on the variational integrators in the system and control community to use in the identification of the second-order mechanical systems. The variational integrator is the solution process of the discrete equation of motion which is derived not from the continuous-time system models but from the discretized version of the Euler-Lagrange equation. The discrete variational integrators were investigated first by Cadzow [9] and then by Logan [10] in control community. An comprehensive study on the variational integrators was made by Marsden and West [11] related to the mechanical systems.

To fix the idea, let $L[z(t), \dot{z}(t)]$ and $f_{L}(t)=$ $f_{L}[z(t), \dot{z}(t)]$ be a Lagrangian and a nonconservative force, where $z(t)$ is the state of the second-order system as the generalized coordinate. Then the Hamilton's principle yields the Euler-Lagrange equation:

$$
\frac{d}{d t}\left(\frac{\partial L(z, \dot{z})}{\partial \dot{z}}\right)-\frac{\partial L(z, \dot{z})}{\partial z}=f_{L}(z, \dot{z}) .
$$

The discrete variational mechanics performs an analogue of this derivation. By approximating the action integral and the external force in some sense, the Hamilton's principle yields the discrete Euler-Lagrange equation in the form: [11] 


$$
\begin{aligned}
& D_{2} L_{d}\left(z_{k-1}, z_{k}\right)+D_{1} L_{d}\left(z_{k}, z_{k+1}\right) \\
& \quad+f_{d}^{+}\left(z_{k-1}, z_{k}\right)+f_{d}^{-}\left(z_{k}, z_{k+1}\right)=0
\end{aligned}
$$

in which $z_{k}=z\left(t_{k}\right) ; L_{d}\left(z_{k-1}, z_{k}\right)$ is the discrete Lagrangian obtained by approximating the action integral along the curve segment between $z_{k-1}$ and $z_{k} ; D_{i}$ $(i=1,2)$ is the partial differential operator acting on the $i$ th argument of the function; and $f_{d}^{ \pm}(\cdot, \cdot)$ are approximate forms concerned with the force $f_{L}(z, \dot{z})$. In [11] the force is pressumed as being nonrandom.

In this paper, taking into account that the system is subjected to a random input, the author will reinvestigate the discrete mechanics from the stochastic point of view based on the Nelson's stochastic mechanics, and consider the identification problem of the structural systems.

\section{Nelson Process and Stochastic Calculus of Variations}

Consider a vector second-order system:

$$
M \ddot{z}(t)+C \dot{z}(t)+K z(t)=G w(t)
$$

in which the matrices $M, C, K$ are the mass, damping and stiffness, respectively; $z(t)=\left[z_{1}(t), z_{2}(t)\right.$, $\left.\cdots, z_{n}(t)\right]^{\mathrm{T}}$ and $w(t)=\left[w_{1}(t), w_{2}(t), \cdots, w_{n}(t)\right]^{\mathrm{T}}$ (the superscript $\mathrm{T}$ denotes transpose) are $n$-vector displacement and random excitation input; and $G$ is a known matrix. For examples of such mechanical or structural systems, refer [1]-[6] and [12]. If we regard $z_{i}(t)$ and $w_{i}(t)$ as the transverse displacement from its equilibrium state and the external random load at the $i$ th floor of an $n$-story building, then (3) is the typical dynamics of $n$-story bending-shear model of high-rise buildings subjected to the random load such as wind pressure [13], [14]. The matrices $M$ and $K$ are symmetric positive-definite, while $C$ is symmetric positivesemidefinite; but they are all unknown. Our problem is to identify these matrices from the sampled observation data on $z(t)$, or $\dot{z}(t)$ or $\ddot{z}(t)$.

The input $w(t)$ is random, so it should be emphasized that the output $z(t)$ becomes inevitably a random process. So, the expressions $\dot{z}(t)$ and $\ddot{z}(t)$ in $(3)$ are quite formal and have no longer their meanings as velocity and acceleration in the ordinary sense. To remedy such an inadequate situation, we introduce here the Nelson process for the output $z(t)$..

Let $(\Omega, \mathcal{A}, P)$ be a base probability space. A mapping $z$ from a time interval $T$ into a Hilbert space $H=L_{2}\left((\Omega, P) ; R^{n}\right)$ is a stochastic process in $R^{n}$ if $t \mapsto z(t)$ is continuous from $T$ into $H$. We consider two filtrations (viz., increasing families of $\sigma$-algebras) $\mathcal{P}_{t}$ and $\mathcal{F}_{t}$ with $\mathcal{P}_{s} \subset \mathcal{P}_{t}$ and $\mathcal{F}_{s} \subset \mathcal{F}_{t}$ for $s \leq t$ to which $z$ is adapted. A class of stochastic processes having two mean velocities

$$
\begin{aligned}
& D z(t)=\lim _{h \downarrow 0} \mathcal{E}\left\{\frac{z(t+h)-z(t)}{h} \mid \mathcal{P}_{t}\right\} \\
& D_{*} z(t)=\lim _{h \downarrow 0} \mathcal{E}\left\{\frac{z(t)-z(t-h)}{h} \mid \mathcal{F}_{t}\right\},
\end{aligned}
$$

where $\mathcal{E}\{\cdot \mid \cdot\}$ denotes the conditional expectation, was discovered by Nelson [15], [16] and is called the Nelson process [17]. The conditions in (4) and (5) may be replaced by the realization $z(t)=z$ at the present time $t$. If $z(t)$ is a smooth function of $t$, these two velocities coincide, and $D z(t)=D_{*} z(t)=d z(t) / d t$. These two velocities are called the mean forward or mean backward velocity (derivative), respectively.

Let the output of a second-order system, $z(t)$, be a Nelson process on $[0, T]$ and let $L$ be a scalar function as Lagrangian [18]

$$
L=L\left[z(t), D z(t), D_{*} z(t)\right] .
$$

Furthermore, let $W(t)$ be the work done by a nonconservative force $f_{L}(t)=f_{L}\left[t, D z(t), D_{*} z(t)\right]$. Then, consider a functional defined by

$$
\begin{array}{r}
J[z]=\mathcal{E}\left\{\int_{0}^{T} L\left[z(t), D z(t), D_{*} z(t)\right] d t\right. \\
\left.+\int_{0}^{T} W(t) d t\right\} .
\end{array}
$$

The Hamilton's principle [19], $\delta J[z]=0$, is expressed as

$$
\begin{aligned}
\mathcal{E}\left\{\delta \int_{0}^{T} L\left[z(t), D z(t), D_{*} z(t)\right] d t\right. & \\
& \left.+\int_{0}^{T} \delta W(t) d t\right\}=0
\end{aligned}
$$

so that we have

$$
\begin{aligned}
& 0=\int_{0}^{\mathrm{T}} \mathcal{E}\left\{\left(\frac{\partial L}{\partial z}\right)^{\mathrm{T}} \delta z(t)+\left(\frac{\partial L}{\partial D z}\right)^{\mathrm{T}} \delta(D z(t))\right. \\
& \left.+\left(\frac{\partial L}{\partial D_{*} z}\right)^{\mathrm{T}} \delta\left(D_{*} z(t)\right)\right\} d t+\mathcal{E}\left\{\int_{0}^{T} \delta W(t) d t\right\} .
\end{aligned}
$$

The virtual work $\delta W(t)$ in (9) is given by

$$
\delta W(t)=f_{L}^{\mathrm{T}}\left(t, D z, D_{*} z\right) \delta z(t) .
$$

In order to calculate the first integral in (9), we need the following Nelson's lemma.

Lemma (Nelson [16], [20]) Let $f(\xi(t))$ and $g(\xi(t))$ be vector-valued functions of a Nelson process $\xi(t)$. Then,

$$
\begin{gathered}
\int_{0}^{T} \mathcal{E}\left\{D f^{\mathrm{T}}(\xi(t)) g(\xi(t))\right\} d t \\
=\mathcal{E}\left\{f^{\mathrm{T}}(\xi(T)) g(\xi(T))-f^{\mathrm{T}}(\xi(0)) g(\xi(0))\right\} \\
-\int_{0}^{T} \mathcal{E}\left\{f^{\mathrm{T}}(\xi(t)) D_{*} g(\xi(t))\right\} d t
\end{gathered}
$$


This is the integration by parts formula for the Nelson process. A brief sketch of the proof is given in [21].

Noting $\delta(D z(t))=D(\delta z(t))$ and identifying $\delta z(t)$ and $\partial L / \partial D z$ as $f(\xi(t))$ and $g(\xi(t))$, respectively, in (11), we have for the middle term in the first integral in (9) that

$$
\begin{aligned}
\int_{0}^{T} \mathcal{E}\left\{\left(\frac{\partial L}{\partial D z}\right)^{\mathrm{T}} \delta(D z(t))\right\} d t \\
=\int_{0}^{T} \mathcal{E}\left\{\left(\frac{\partial L}{\partial D z}\right)^{\mathrm{T}} D(\delta z(t))\right\} d t \\
=\mathcal{E}\left\{\left.\left(\frac{\partial L}{\partial D z}\right)^{\mathrm{T}} \delta z(t)\right|_{t=0} ^{t=T}\right\} \\
-\int_{0}^{T} \mathcal{E}\left\{(\delta z(t))^{\mathrm{T}} D_{*}\left(\frac{\partial L}{\partial D z}\right)\right\} d t \\
=-\int_{0}^{T} \mathcal{E}\left\{D_{*}^{\mathrm{T}}\left(\frac{\partial L}{\partial D z}\right) \delta z(t)\right\} d t
\end{aligned}
$$

since $\delta z(t)=0$ at $t=0$ and $T$. Similarly, we obtain

$$
\begin{aligned}
\int_{0}^{T} \mathcal{E} & \left\{\left(\frac{\partial L}{\partial D_{*} z}\right)^{\mathrm{T}} \delta\left(D_{*} z(t)\right)\right\} d t \\
& =-\int_{0}^{T} \mathcal{E}\left\{D^{\mathrm{T}}\left(\frac{\partial L}{\partial D_{*} z}\right) \delta z(t)\right\} d t
\end{aligned}
$$

Therefore, (9) becomes

$$
\begin{aligned}
& 0=\int_{0}^{T} \mathcal{E}\left\{\left[\frac{\partial L}{\partial z}-D_{*}\left(\frac{\partial L}{\partial D z}\right)\right.\right. \\
&\left.\left.-D\left(\frac{\partial L}{\partial D_{*} z}\right)\right]^{\mathrm{T}} \delta z(t) d t\right\} \\
&+\mathcal{E}\left\{\int_{0}^{T} f_{L}^{T}\left(t, D z, D_{*} z\right) \delta z(t) d t\right\} \\
&=\mathcal{E}\left\{\int _ { 0 } ^ { T } \left[\frac{\partial L}{\partial z}-D_{*}\left(\frac{\partial L}{\partial D z}\right)-D\left(\frac{\partial L}{\partial D_{*} z}\right)\right.\right. \\
&+\left.\left.f_{L}\left(t, D z, D_{*} z\right)\right]^{\mathrm{T}} \delta z(t) d t\right\} .
\end{aligned}
$$

Theorem: A necessary and sufficient condition for a process $z(t)$ subjected to a force $f_{L}(t)=f_{L}\left(t, D z, D_{*} z\right)$ to be a stationary point of the functional $J[z]$ is that

$$
\begin{aligned}
& D\left(\frac{\partial L}{\partial D_{*} z}\right)\left(z, D z, D_{*} z\right) \\
& \quad+D_{*}\left(\frac{\partial L}{\partial D z}\right)\left(z, D z, D_{*} z\right) \\
& \quad-\frac{\partial L}{\partial z}\left(z, D z, D_{*} z\right)=f_{L}\left(t, D z, D_{*} z\right) .
\end{aligned}
$$

Proof: Since the variation $\delta z(t)(0<t<T)$ is arbitrary, (13) is obvious from (12). (QED)
This theorem is an extension of the theorem obtained by Yasue [18] to nonconservative systems, and (13) is a stochastic counterpart of the classical (forced) Euler-Lagrange equation (1). We call (13) the (forced) stochastic Euler-Lagrange equation.

\section{Discrete Structural Systems}

For the postulated system model (3), consider the Lagrangian,

$$
\begin{aligned}
L\left(z, D z, D_{*} z\right)= & T\left(D z, D_{*} z\right)-V(z) \\
=\frac{1}{2}\left\{\frac{1}{2}(D z)^{\mathrm{T}}\right. & \left.M(D z)+\frac{1}{2}\left(D_{*} z\right)^{\mathrm{T}} M\left(D_{*} z\right)\right\} \\
& -\frac{1}{2} z^{\mathrm{T}} K z
\end{aligned}
$$

where $T\left(D z, D_{*} z\right)$ and $V(z)$ are kinetic and potential energies. Further, let the nonconservative force (called the Lagrangian force) $f_{L}(t)$ consist of the dissipation force $f_{D}(t)$ (which corresponds to $-C \dot{z}(t)$ ) and the random external force $G w(t)$,

$$
f_{L}(t)=-C \frac{1}{2}\left\{D z(t)+D_{*} z(t)\right\}+G w(t) .
$$

Then, by imposing the process $z(t)$ to be a stationary point of the functional $J[z]$ under (14) and (15), the stochastic Euler-Lagrange equation (13) yields

$$
\begin{aligned}
& M \frac{1}{2}\left\{D D_{*} z(t)+D_{*} D z(t)\right\} \\
& \quad+C \frac{1}{2}\left\{D z(t)+D_{*} z(t)\right\}+K z(t)=G w(t) .
\end{aligned}
$$

This is the generalized Newton's equation of motion, so that the expression (3) should be interpreted as (16). Comparing (16) with (3), we know that the formal derivatives $\dot{z}(t)$ and $\ddot{z}(t)$ should be interpreted as

$$
\begin{aligned}
\dot{z}(t) & \sim \frac{1}{2}\left\{D z(t)+D_{*} z(t)\right\} \\
\ddot{z}(t) & \sim \frac{1}{2}\left\{D D_{*} z(t)+D_{*} D z(t)\right\} .
\end{aligned}
$$

Though the expression (16) is rigorous, the formal equation of motion (3) is rather more intuitive than (16).

Let us proceed to obtain the discrete form of (16). To do this, $N$ equi-partition the interval $[0, T]$ and denote as $z_{k}=z\left(t_{k}\right)=z(k h)(h=T / N ; k=0,1,2, \cdots, N-1)$. Then, approximate as

$$
D z\left(t_{k}\right) \cong \frac{z_{k+1}-z_{k}}{h}, D_{*} z\left(t_{k}\right) \cong \frac{z_{k}-z_{k-1}}{h} .
$$

Then,

$$
\begin{aligned}
& \frac{1}{2}\left\{D z\left(t_{k}\right)+D_{*} z\left(t_{k}\right)\right\}=\frac{1}{2 h}\left(z_{k+1}-z_{k-1}\right) \\
& \frac{1}{2}\left\{D D_{*} z\left(t_{k}\right)+D_{*} D z\left(t_{k}\right)\right\} \\
& \quad=\frac{1}{h^{2}}\left(z_{k+1}-2 z_{k}+z_{k-1}\right)
\end{aligned}
$$

and hence (16) is discretized as 


$$
\begin{aligned}
\left(\frac{M}{h}+\right. & \left.\frac{C}{2}\right) z_{k+1}+\left(-\frac{2 M}{h}+h K\right) z_{k} \\
& +\left(\frac{M}{h}-\frac{C}{2}\right) z_{k-1}=h G w(k h)
\end{aligned}
$$

or

$$
M_{d} z_{k+2}+C_{d} z_{k+1}+K_{d} z_{k}=G w_{k+1},
$$

where $w_{k+1}=h w((k+1) h)$ and

$$
\left\{\begin{array}{l}
M_{d}=\frac{M}{h}+\frac{C}{2}, \quad C_{d}=-\frac{2 M}{h}+h K \\
K_{d}=\frac{M}{h}-\frac{C}{2} .
\end{array}\right.
$$

We call (22) the discrete structural dynamics as a discrete-time counterpart of continuous system (3). It should be noted that $M_{d}, C_{d}$ and $K_{d}$ in (22) have no their meanings of mass, damping and stiffness.

It is a simple excersise to show the relations

$$
\left\{\begin{array}{l}
M=\frac{h}{2}\left(M_{d}+K_{d}\right), \quad C=M_{d}-K_{d} \\
K=\frac{1}{h}\left(M_{d}+C_{d}+K_{d}\right) .
\end{array}\right.
$$

\section{Another Derivation of Discrete Structural Systems}

Though the disrete structural dynamics (22) has been derived by discretizing the forced stochastic EulerLagrange equation (13), it can be derived directly from the Hamilton's principle (8), i.e.

$$
\begin{aligned}
& \mathcal{E}\left\{\int_{0}^{T} \delta L\left[z(t), D z(t), D_{*} z(t)\right] d t\right. \\
&\left.\quad+\int_{0}^{T} f_{L}^{\mathrm{T}}\left(t, D z, D_{*} z\right) \delta z(t) d t\right\}=0 .
\end{aligned}
$$

A necessary and sufficient condition is that

$$
\begin{aligned}
\int_{0}^{T} & \delta L\left[z(t), D z(t), D_{*} z(t)\right] d t \\
& \quad+\int_{0}^{T} f_{L}^{\mathrm{T}}\left(t, D z, D_{*} z\right) \delta z(t) d t=0 .
\end{aligned}
$$

This condition must hold pathwise along $z(t)$-process.

Now, recalling the definitions $D z(t)$ and $D_{*} z(t)$, approximate the integral $\int_{0}^{T} \delta L\left(z, D z, D_{*} z\right) d t$ by the discrete sum evaluated along the curve of points $\left\{z_{k}\right\}_{k=0,1, \cdots, N}$,

$$
\sum_{k=0}^{N-1} \delta L\left[z\left(t_{k}\right), D z\left(t_{k}\right), D_{*} z\left(t_{k}\right)\right] h+o(h)
$$

in which

$$
\begin{aligned}
& L\left[z\left(t_{k}\right), D z\left(t_{k}\right), D_{*} z\left(t_{k}\right)\right] h \\
& \quad=h L\left(z_{k}, \frac{z_{k+1}-z_{k}}{h}, \frac{z_{k}-z_{k-1}}{h}\right) \\
& \quad=: L_{d}\left(z_{k-1}, z_{k}, z_{k+1}\right) .
\end{aligned}
$$

Hence, the discrete sum of variations (27) becomes

$$
\begin{aligned}
\sum_{k=0}^{N-1} & \delta L_{d}\left(z_{k-1}, z_{k}, z_{k+1}\right) \\
= & \sum_{k=0}^{N-1}\left[\left(\frac{\partial L_{d}\left(z_{k-1}, z_{k}, z_{k+1}\right)}{\partial z_{k-1}}\right)^{\mathrm{T}} \delta z_{k-1}\right. \\
& +\left(\frac{\partial L_{d}\left(z_{k-1}, z_{k}, z_{k+1}\right)}{\partial z_{k}}\right)^{\mathrm{T}} \delta z_{k} \\
& \left.+\left(\frac{\partial L_{d}\left(z_{k-1}, z_{k}, z_{k+1}\right)}{\partial z_{k+1}}\right)^{\mathrm{T}} \delta z_{k+1}\right] \\
= & \sum_{k=0}^{N-1}\left[D_{1} L_{d}\left(z_{k}, z_{k+1}, z_{k+2}\right)\right. \\
& +D_{2} L_{d}\left(z_{k-1}, z_{k}, z_{k+1}\right) \\
& \left.+D_{3} L_{d}\left(z_{k-2}, z_{k-1}, z_{k}\right)\right]^{\mathrm{T}} \delta z_{k} \\
& -\left[D_{1} L_{d}\left(z_{N-1}, z_{N}, z_{N+1}\right)\right]^{\mathrm{T}} \delta z_{N-1},
\end{aligned}
$$

where $D_{i}(i=1,2,3)$ is the partial differential operator acting on the $i$ th argument, for instance, $D_{2} L_{d}(a, b, c)=\partial L_{d}(a, b, c) / \partial b$, and we have rearranged the summations by $\delta z_{k}$ and used the constraints $\delta z_{-1}=$ $\delta z_{0}=\delta z_{N}=0$.

On the other hand, applying the approximations to the stochastic derivatives $D z(t)$ and $D_{*} z(t)$ in the Lagrangian force $f_{L}(t)=f_{L}\left(t, D z, D_{*} z\right)$ given by (15), we obtain

$$
\begin{aligned}
& f_{L}\left(t_{k}, D z_{k}, D_{*} z_{k}\right) \\
& \quad=-\frac{1}{2} C\left(\frac{z_{k+1}-z_{k}}{h}+\frac{z_{k}-z_{k-1}}{h}\right)+G w(k h) \\
& \quad=-\frac{1}{2} C\left(\frac{z_{k+1}-z_{k-1}}{h}\right)+G w(k h) .
\end{aligned}
$$

Hence, the second integral in the L.H.S. of (26) is approximated as

$$
\int_{0}^{T} f_{L}^{\mathrm{T}}\left(t, D z, D_{*} z\right) \delta z(t) d t=\sum_{k=0}^{N-1} f_{d}^{\mathrm{T}}(k h) \delta z_{k}+o(h),
$$

where

$$
f_{d}(k h)=h f_{L}\left(t_{k}, D z_{k}, D_{*} z_{k}\right) .
$$

Therefore, the condition (26) yields its approximate form: 


$$
\begin{aligned}
0= & \sum_{k=0}^{N-1}\left[D_{1} L_{d}\left(z_{k}, z_{k+1}, z_{k+2}\right)\right. \\
+ & D_{2} L_{d}\left(z_{k-1}, z_{k}, z_{k+1}\right) \\
& \left.+D_{3} L_{d}\left(z_{k-2}, z_{k-1}, z_{k}\right)+f_{d}(k h)\right]^{\mathrm{T}} \delta z_{k} \\
& \quad-\left[D_{1} L_{d}\left(z_{N-1}, z_{N}, z_{N+1}\right)\right]^{\mathrm{T}} \delta z_{N-1}
\end{aligned}
$$

from which we obtain the second-oredr (forced) discrete Euler-Lagrange equation

$$
\begin{gathered}
D_{3} L_{d}\left(z_{k-2}, z_{k-1}, z_{k}\right)+D_{2} L_{d}\left(z_{k-1}, z_{k}, z_{k+1}\right) \\
\quad+D_{1} L_{d}\left(z_{k}, z_{k+1}, z_{k+2}\right)+f_{d}(k h)=0
\end{gathered}
$$

with constraint

$$
D_{1} L_{d}\left(z_{N-1}, z_{N}, z_{N+1}\right)=0
$$

since all variations $\left\{\delta z_{k}\right\}$ are arbitrary. Equation (34) is the natural boundary condition, and (33) corresponds to $(2)$.

For the Lagrangian (14) the discrete Lagrangian $L_{d}$ is given by

$$
\begin{aligned}
& L_{d}\left(z_{k-1}, z_{k}, z_{k+1}\right) \\
&=h {\left[\frac{1}{4}\left(\frac{z_{k+1}-z_{k}}{h}\right)^{\mathrm{T}} M\left(\frac{z_{k+1}-z_{k}}{h}\right)\right.} \\
&+ \frac{1}{4}\left(\frac{z_{k}-z_{k-1}}{h}\right)^{\mathrm{T}} M\left(\frac{z_{k}-z_{k-1}}{h}\right) \\
&\left.-\frac{1}{2} z_{k}^{\mathrm{T}} K z_{k}\right]
\end{aligned}
$$

so that we compute

$$
\begin{gathered}
D_{1} L_{d}\left(z_{k}, z_{k+1}, z_{k+2}\right)=\frac{\partial}{\partial z_{k}} L_{d}\left(z_{k}, z_{k+1}, z_{k+2}\right) \\
=-\frac{1}{2} M\left(\frac{z_{k+1}-z_{k}}{h}\right) \\
D_{2} L_{d}\left(z_{k-1}, z_{k}, z_{k+1}\right) \\
=-\frac{1}{2} M\left(\frac{z_{k+1}-2 z_{k}+z_{k-1}}{h}\right)-h K z_{k} \\
D_{3} L_{d}\left(z_{k-2}, z_{k-1}, z_{k}\right)=\frac{1}{2} M\left(\frac{z_{k}-z_{k-1}}{h}\right) .
\end{gathered}
$$

Also, for the Lagrangian force (15)

$$
f_{d}(k h)=-\frac{1}{2} C\left(z_{k+1}-z_{k-1}\right)+h G w(k h) .
$$

Therefore, the discrete Euler-Lagrange equation (33) is expressed as

$$
\begin{aligned}
0= & \frac{1}{2} M\left(\frac{z_{k}-z_{k-1}}{h}\right) \\
& -\frac{1}{2} M\left(\frac{z_{k+1}-2 z_{k}+z_{k-1}}{h}\right)-h K z_{k} \\
& -\frac{1}{2} M\left(\frac{z_{k+1}-z_{k}}{h}\right)-\frac{1}{2} C\left(z_{k+1}-z_{k-1}\right) \\
& +h G w(k h) .
\end{aligned}
$$

By shifting the time $k$ to $k+1$, we obtain the discrete structural dynamics (22).

The idea of discretization of the action integral was tried first by Nobel laureate Richard Feynman (1948) to derive his path integrals in quantum physics [22].

\section{Identification of Structural Matrices}

The identification of structural matrices $(M, C, K)$ can be performed by estimating $\left(M_{d}, C_{d}, K_{d}\right)$ of the discrete structural dynamics (22) using the relation (24). We employ the so-called subspace identification approach. To do this, assume that $w(t)$ is sufficiently rich for identification and also that we have acquired the input and output data, $\{w(k h)\}_{k=0,1, \cdots, N-1}$ and $\{y(k h)\}_{k=0,1, \cdots, N-1}$, over a suitably long time interval with sampling period $h$. Otherwise, we input a persistently exciting random force to the structural system.

In this section, two cases of measurement scheme on $z(t)$ are considered: the first is the case where only the displacement $z(t)$ is measured, while the other not only $z(t)$ but also the velocity $\dot{z}(t)$ and/or acceleration $\ddot{z}(t)$ can be observed.

\section{(i) Measurement Scheme 1}

For simplicity, assume that we can take $n$-vector output data $y\left(t_{k}\right) \in R^{n}$ on the displacement $z_{k}=z(k h)$ with additive noise such that

$$
y_{k}=H z_{k}+v_{k},
$$

where $v_{k}$ is the stationary white noise sequence, and $H$ is a nonsingular $n \times n$-matrix. Then, we have

$$
z_{k}=H^{-1}\left\{y_{k}-v_{k}\right\}
$$

The assumption that the dimension of $y_{k}$ is the same as the stories of the building structure may seem peculiar; however, this will be affirmable from the practical point of view because sensors for measurering vibrations become inexpensive nowadays. Otherwise, use the generalized inverse insted of $\mathrm{H}^{-1}$.

Substituting (38) into the discrete structural dynamics (22), we have the following regression model:

$$
M_{d} \hat{y}_{k+2}+C_{d} \hat{y}_{k+1}+K_{d} \hat{y}_{k}=G w_{k+1}+e_{k+2},
$$

where $\hat{y}_{k}=H^{-1} y_{k}$; and

$$
e_{k+2}=M_{d} H^{-1} v_{k+2}+C_{d} H^{-1} v_{k+1}+K_{d} H^{-1} v_{k}
$$


which is regarded as a colored noise sequence.

Here, define matrices

$$
\begin{aligned}
\hat{Y}_{k} & =\left[\begin{array}{llll}
\hat{y}_{k} & \hat{y}_{k+1} & \cdots & \hat{y}_{k+N-1}
\end{array}\right] \in R^{n \times N} \\
W_{k} & =\left[\begin{array}{llll}
w_{k} & w_{k+1} & \cdots & w_{k+N-1}
\end{array}\right] \in R^{n \times N} \\
E_{k} & =\left[\begin{array}{llll}
e_{k} & e_{k+1} & \cdots & e_{k+N-1}
\end{array}\right] \in R^{n \times N} .
\end{aligned}
$$

Then, the regression equation (39) can be expressed in the matrix form,

$$
E_{k}=\left[\begin{array}{llll}
M_{d} & C_{d} & K_{d}-G
\end{array}\right]\left[\begin{array}{l}
\hat{Y}_{k} \\
\hat{Y}_{k-1} \\
\hat{Y}_{k-2} \\
W_{k-1}
\end{array}\right] .
$$

Furthermore, define matrices

$$
A_{0}=\left[\begin{array}{lll}
M_{d} & C_{d} & K_{d}
\end{array}\right], \quad \tilde{Y}_{k}=\left[\begin{array}{c}
\hat{Y}_{k} \\
\hat{Y}_{k-1} \\
\hat{Y}_{k-2}
\end{array}\right]
$$

Then, (41) is expressed as

$$
E_{k}=\left[\begin{array}{ll}
A_{0} & -G
\end{array}\right]\left[\begin{array}{l}
\tilde{Y}_{k} \\
W_{k-1}
\end{array}\right] .
$$

The unknown matrix $A_{0}=\left[M_{d}, C_{d}, K_{d}\right]$ is determined as a least-squares estimate by minimizing the Frobenius norm $\left\|E_{k}\right\|_{F}=\left[\operatorname{tr}\left\{E_{k} E_{k}^{\mathrm{T}}\right\}\right]^{\frac{1}{2}}$. To this end, $L Q$-factorize the last matrix in the R.H.S. of (43) as

$$
\left[\begin{array}{l}
\tilde{Y}_{k} \\
W_{k-1}
\end{array}\right]=\left[\begin{array}{cc}
L_{11} & O \\
L_{21} & L_{22}
\end{array}\right]\left[\begin{array}{l}
Q_{1} \\
Q_{2}
\end{array}\right],
$$

where $L_{11} \in R^{3 n \times 3 n}, L_{21} \in R^{n \times 3 n}, L_{22} \in R^{n \times n}$, and $Q_{1}$ and $Q_{2}$ are orthonormal matrices. Using this factorization, the matrix $E_{k} E_{k}^{\mathrm{T}}$ is evaluated as

$$
\begin{aligned}
& E_{k} E_{k}^{\mathrm{T}}=\left(A_{0} L_{11}-G L_{21}\right)\left(A_{0} L_{11}-G L_{21}\right)^{\mathrm{T}} \\
&+G L_{22} L_{22}^{\mathrm{T}} G^{\mathrm{T}} \\
& \geq G L_{22} L_{22}^{\mathrm{T}} G^{\mathrm{T}},
\end{aligned}
$$

where the equality holds if and only if

$$
A_{0} L_{11}-G L_{21}=0
$$

holds. From this, $\left[M_{d}, C_{d}, K_{d}\right]=A_{0}$ is determined as

$$
\left[\begin{array}{lll}
M_{d} & C_{d} & K_{d}
\end{array}\right]=G L_{21} L_{11}^{-1} \text {. }
$$

Consequently, the structural matrices $(M, C, K)$ can be obtained from (48) using the relation (24). If we define the observation matrix $Y_{k}=$ $\left[y_{k}, y_{k+1}, \cdots, y_{k+N-1}\right]$, then $\hat{Y}_{k}=H^{-1} Y_{k}$. (ii) Measurement Scheme 2

From the physical point of view the direct measurement on the displacement $z(t)$ is rather hard to obtain, especially for the building structures. It will be rather the more practical to use accelerometers for detecting movement of the relevant structure. As well-known, the recent measurement technology develops cheep highperformance accelerometers. So, in this subsection, assuming the use of accelerometers, we investigate the more general case such that

$$
y(t)=H_{a} \ddot{z}(t)+H_{v} \dot{z}(t)+H_{d} z(t)
$$

with some measurement noise, where $y \in R^{n}$ and $H_{a}$, $H_{v}, H_{d}$ are all $n \times n$-matrices. The (formal) velocity $\dot{z}(t)$ and accelration $\ddot{z}(t)$ are discretized according to (17)-(21), so that the discrete version of the observation process $y(t)$ is given by adding the noise term $v(k h)$ as

$$
\begin{aligned}
y(k h)= & \left(\frac{H_{a}}{h^{2}}+\frac{H_{v}}{2 h}\right) z_{k+1}+\left(-\frac{2 H_{a}}{h^{2}}+H_{d}\right) z_{k} \\
& +\left(\frac{H_{a}}{h^{2}}-\frac{H_{v}}{2 h}\right) z_{k-1}+v(k h)
\end{aligned}
$$

or

$$
\bar{y}_{k}=\bar{H}_{a} z_{k+1}+\bar{H}_{v} z_{k}+\bar{H}_{d} z_{k-1}+\bar{v}_{k}
$$

where $\bar{y}_{k}=h y(k h), \bar{v}_{k}=h v(k h)$, and

$$
\left\{\begin{array}{l}
\bar{H}_{a}=\frac{H_{a}}{h}+\frac{H_{v}}{2}, \quad \bar{H}_{v}=-\frac{2 H_{a}}{h}+h H_{d} \\
\bar{H}_{d}=\frac{H_{a}}{h}-\frac{H_{v}}{2} .
\end{array}\right.
$$

From (48)

$$
\bar{y}_{k}=\bar{H}\left[\begin{array}{l}
z_{k+1} \\
z_{k} \\
z_{k-1}
\end{array}\right]+\bar{v}_{k}
$$

where $\bar{H}=\left[\bar{H}_{a}, \bar{H}_{v}, \bar{H}_{d}\right] \in R^{n \times 3 n}$. Using the pseudoinverse $\bar{H}^{\dagger}=\bar{H}^{\mathrm{T}}\left(\bar{H} \bar{H}^{\mathrm{T}}\right)^{-1}$, we derive

$$
\left[\begin{array}{l}
z_{k+1} \\
z_{k} \\
z_{k-1}
\end{array}\right]=\bar{H}^{\dagger}\left\{\bar{y}_{k}-\bar{v}_{k}\right\}
$$

Since (22) is expressed as

$$
A_{0}\left[\begin{array}{l}
z_{k+1} \\
z_{k} \\
z_{k-1}
\end{array}\right]=G w_{k}
$$

we have from (51) and (52) that

$$
A_{0} \hat{y}_{k}=G w_{k}+e_{k},
$$

where $\hat{y}_{k}=\bar{H}^{\dagger} \bar{y}_{k}$ and $e_{k}=A_{0} \bar{H}^{\dagger} \bar{v}_{k}$. 
Here, define matrices $\hat{Y}_{k} \in R^{3 n \times N}, W_{k}, E_{k} \in R^{n \times N}$ for $\hat{y}_{k}, w_{k}$ and $e_{k}$ similarly as done in Case (i). Then, we have from (53) that

$$
A_{0} \hat{Y}_{k}=G W_{k}+E_{k}
$$

or

$$
E_{k}=\left[\begin{array}{ll}
A_{0} & -G
\end{array}\right]\left[\begin{array}{l}
\hat{Y}_{k} \\
W_{k}
\end{array}\right]
$$

which corresponds to (42) for Case (i). Using the $L Q$ factorization

$$
\left[\begin{array}{l}
\hat{Y}_{k} \\
W_{k}
\end{array}\right]=\left[\begin{array}{cc}
\hat{L}_{11} & O \\
\hat{L}_{21} & \hat{L}_{22}
\end{array}\right]\left[\begin{array}{l}
\hat{Q}_{1} \\
\hat{Q}_{2}
\end{array}\right],
$$

the unknown matrix $A_{0}=\left[M_{d}, C_{d}, K_{d}\right]$ is determined as

$$
\left[\begin{array}{lll}
M_{d} & C_{d} & K_{d}
\end{array}\right]=G \hat{L}_{21} \hat{L}_{11}^{-1}
$$

\section{Discussions}

(i) Using the idea of variational integrators which was developed comprehensively by Marsden and West [11], Bruschetta, et al. [8] have investigated a discretization technique of the Lagrange equations of motion for mechanical systems. Based on the discrete EulerLagrange equation (2), Bruschetta et al. have established an identification method using the midpoint rule, $z(t) \cong\left(z_{k+1}+z_{k}\right) / 2$ and $\dot{z}(t) \cong\left(z_{k+1}-z_{k}\right) / h$. This is the big different point from the author's in evaluating Lagrange function and Lagrange force. Their discrete structural dynamics which correspond to (22) is given by

$$
M_{d} z_{k+2}+C_{d} z_{k+1}+K_{d} z_{k}=G w_{k+2}
$$

with

$$
\begin{aligned}
& M_{d}=\frac{M}{h}+\frac{C}{2}+\frac{h K}{4}, \quad C_{d}=-\frac{2 M}{h}+\frac{h K}{2} \\
& K_{d}=\frac{M}{h}-\frac{C}{2}+\frac{h K}{4} \\
& w_{k+2}=\frac{h}{4}\{w(k h)+2 w((k+1) h)+w((k+2) h)\}
\end{aligned}
$$

which are slightly different from the author's (see (22) and (23) for comparison).

The difference between theirs and the author's seem to have come mainly from the facts that (i) our discrete Lagrangian in (33) is evaluated by three points

\begin{tabular}{|c|c|}
\hline APPROACH & $(M, C, K) \quad$ AND $\quad w^{*}$ \\
\hline $\begin{array}{l}\text { Stochastic } \\
\text { Calculus }\end{array}$ & $\begin{aligned} M & =\frac{h}{2}\left(M_{d}+K_{d}\right) \\
C & =M_{d}-K_{d} \\
K & =\frac{1}{h}\left(M_{d}+C_{d}+K_{d}\right) \\
w^{*} & =h w((k+1) h)\end{aligned}$ \\
\hline $\begin{array}{l}\text { Variational } \\
\text { Integrators } \\
\text { (Bruschetta } \\
\text { et al., 2009) }\end{array}$ & $\begin{aligned} M & =\frac{h}{4}\left(M_{d}-C_{d}+K_{d}\right) \\
C & =M_{d}-K_{d} \\
K & =\frac{1}{h}\left(M_{d}+C_{d}+K_{d}\right) \\
w^{*}=\frac{h}{4}\{w(k h)+2 w((k+1) h) & +w((k+2) h)\}\end{aligned}$ \\
\hline $\begin{array}{c}\text { Ordinary } \\
\text { Differences }\end{array}$ & $\begin{aligned} M & =h M_{d} \\
C & =2 M_{d}+C_{d} \\
K & =\frac{1}{h}\left(M_{d}+C_{d}+K_{d}\right) \\
w^{*} & =h w(k h)\end{aligned}$ \\
\hline
\end{tabular}
$z_{k-1}, z_{k}$ and $z_{k+1}$, while Bruschetta et al.'s use only two points $z_{k}$ and $z_{k+1}$ according to the midpoint rule, so that there does not appear the differential term $D_{3}$, and that (ii) in the Bruchetta et al.'s method, the Lagrangian force is averaged at three instants $t_{k}, t_{k+1}$ and $t_{k+2}$ as shown above in $w_{k+2}$, while in the author's it is
Table 1. Comparison of identification results.

evaluated only at $t_{k+1}$. Although in Bruschetta et al. they do not refer any thing about the input $w(t)$ in $(3)$ whether it is random or nonrandom, it should be especially considered when any approximation is needed that the external input is a random process or a deterministic one. According to the result of Bruschetta et al., even a random input $w(t)$ it is "averaged" by three points.

Assuming that the continuous-time structural model (3) is approximated in the form,

$$
M_{d} z_{k+2}+C_{d} z_{k+1}+K_{d} z_{k}=G w^{*}
$$

comparisons of the identification results of the author's based on stochastic calculus and theirs are listed in Table 1 .

(ii) Perhaps, the most typical and conventional method to obtain the discrete-time vesions for the continuous-time structural dynamics is the direct application of differential quotiens, $\dot{z}(t) \cong\left(z_{k+1}-z_{k}\right) / h$ and $\ddot{z}(t) \cong\left(z_{k+2}-2 z_{k+1}+z_{k}\right) / h^{2}$, to the dynamics $(3)$. This approach yields the form of (57) with

$$
\begin{aligned}
M_{d} & =\frac{M}{h}, \quad C_{d}=-\frac{2 M}{h}+C \\
K_{d} & =\frac{M}{h}-C+h K \\
w^{*} & =h w(k h) .
\end{aligned}
$$

We refer to this approach as the ordinary differences and the identification result is listed also in Table 1. 
(iii) From the discussion in Section 3, we know that the result shown in Table 1 as "stochastic calculus" can be obtained by replacing derivatives $\dot{z}(t)$ and $\ddot{z}(t)$ in the formal equation of motion (3) by their stochastic derivatives (17) and (18) and by applying their discretizations (20) and (21).*

\section{Conclusions}

Based on the Nelson process, a discrete second-order model has been derived for a class of stochastic structural or mechanical systems which are subjected to random disturbance input. Two approaches to derive the discrete models have been shown: one of which is the discretization from the stochastic Euler-Lagnrange equation, while the other the direct discretization of the Hamilton's principle. The stochastic mechanics from the Hamiltonian viewpoint and the discretization of the stochastic Hamiltonian system are investigated in [21].

\section{References}

[1] M. Abdelghani, M. Verhaegen, P. Van Overschee, and B. De Moor: Comparison Study of Subspace Identification Methods Applied to Flexible Structures, Mechanical Systems and Signal Processing, 12-5, 679/692 (1998).

[2] C. S. Huang: Structural Identification from Ambient Vibration Measurement Using the Multivariate AR Model, J. of Sound and Vibration, 241-3, $337 / 359$ (2001).

[3] M. De Angelis, H. Luş, R. Betti, and R. W. Longman: Extracting Physical Parameters of Mechanical Models From Identified State-Space Representations, Trans. ASME, J. of Applied Mechanics, 69, $617 / 625$ (2002).

[4] H. Luş, M. De Angelis, R. Betti, and R. W. Longman: Constructing Second-Order Models of Mechanical Systems from Identified State Space Realizations; Part I: Theoretical Discussions, J. of Engineering Mechanics, 129-5, 477/488 (2003).

[5] C.-F. Hung, W.-J. Ko, and Y.-T. Peng: Identification of Modal Parameters from Measured Input and Output Data Using a Vector Backward AutoRegressive with Exogeneous Model, J. of Sound and Vibration, 276, 1043/1063 (2004).

[6] K. Sawada, K. Kameyama, S. Fujikawa, and A. Ohsumi: Estimation of Modal Parameters of a Multi-story Structure via Continuous-time 4SID Method, Proc. 48th Joint Automatic Control Conf., Nagano, 811/814 (2005) (in Japanese).

*In [23] the author referred to this method as the stochastic-derivative approximation; however, unfortunately, the result obtained was wrong by simple mistake in calculation.
[7] L. Dieci and A. Papini: Conditioning and Padé Approximation of the Logarithm of a Matrix, SIAM J. Mart. Anal. Appl., 21, 913/930 (2000).

[8] M. Bruschetta, G. Picci, and A. Saccon: Discrete Mechanical Systems: Second Order Modelling and Identification, Preprints of the 15th IFAC Symp. on System Identification (SYSID 2009), SaintMalo, France, 456/461 (2009).

[9] J. A. Cadzow: Discrete Calculus of Variations, Int. J. Control, 11-3, 393/407 (1970).

[10] J. D. Logan: First Integrals in the Discrete Variational Calculus, Aequationes Mathematicae, 9, 210/220 (1973).

[11] J. E. Marsden and M. West: Discrete Mechanics and Variational Integrators, Acta Numerica, Cambridge Univ. Press, 357/514 (2001).

[12] R. E. Skelton: Dynamic Systems Control: Linear Systems Analysis and Synthesis, John Wiley \& Sons, New York (1988).

[13] J. Minato and A. Ohsumi: Control for High-rise Buildings Subjected to Wind and Seismic Disturbances, Proc. of SICE Annual Conf. 2003 in Fukui, Fukui, 2020/2024 (2003).

[14] J. Minato, A. Ohsumi, and Y. Sawada: Generation of Artificial Wind and Estimation of Load from Structural Response, Int. J. of Innovative Computing, Information and Control, 6-10, 4615/4629 (2010).

[15] E. Nelson: Derivation of the Schrödinger Equation from Newtonian Mechnaics, Physical Review, 1504, 1079/1085 (1966).

[16] E. Nelson: Dynamical Theories of Brownian Motion, Princeton Univ. Press, Princeton, New Jersey (1967).

[17] J.-C. Zambrini: Stochastic Dynamics: A Review of Stochastic Calculus of Variations, Int. J. of Theoretical Physics, 24-3, 277/327 (1985).

[18] K. Yasue: Stochastic Calculus of Variations, J. of Funct. Anal., 41, 327/340 (1981).

[19] E. T. Whittaker: A Treatise on the Analytical Dynamics of Particles and Rigid Bodies, 4th Edition, Cambridge Univ. Press, Cambridge (1937).

[20] E. Nelson: Quantum Fluctuations, Princeton Univ. Press, Princeton, New Jersey (1985).

[21] A. Ohsumi: Modeling of Second-Order Discrete Systems from Stochastic Calculus of Variations, Proc. 10th SICE Conf. on Control, Kumamoto, CD-ROM (2010).

[22] R. P. Feynman: Space-Time Approach to NonRelativistic Quantum Mechanics, Rev. Mod. Phys., 20-2, 367/387 (1948).

[23] A. Ohsumi: Discrete Calculation of Variations via Stochastic Mechanics and Subspace Identification of Second-Order Systems, Proc. 10th SICE Conf. on Control, Kumamoto, CD-ROM (2010). 